(C) 2016. This manuscript version is made available under the CC-BY-NC-ND 4.0 license http://creativecommons.org/licenses/by-nc-nd/4.0/

\title{
Pointwise aggregation of maps: its structural functional equation and some uses in social choice theory
}

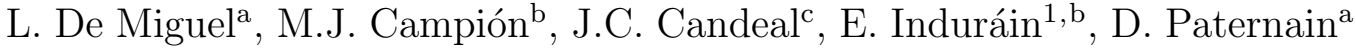 \\ ${ }^{a}$ Instituto de Investigación SmartCities and Departamento de Automática y \\ Computación, Universidad Pública de Navarra, 31006 Pamplona, Spain \\ ${ }^{b}$ Instituto de Investigación InaMat and Departamento de Matemáticas, Universidad \\ Pública de Navarra, 31006 Pamplona, Spain \\ ${ }^{c}$ Departamento de Análisis Económico, Universidad de Zaragoza, 50009 Zaragoza, Spain
}

\begin{abstract}
We study a structural functional equation that is directly related to the pointwise aggregation of a finite number of maps from a nonempty given set into another. First we establish links between pointwise aggregation and invariance properties. Then, paying attention to the particular case of aggregation operators of a finite number of real-valued functions, we characterize several special kinds of aggregation operators, as strictly monotone modifications of projections. As a case study, we introduce a first approach of type-2 fuzzy sets via fusion operators. We develop some applications and possible uses related to the analysis of properties of social evaluation functionals in social choice, showing that those functionals can actually be described by using methods that derive from this setting.
\end{abstract}

Keywords: Aggregation operators, functional equations, pointwise aggregation, real-valued functions, type-2 fuzzy sets, applications to social choice.

2000 MSC: 39B52, 03E72, 26A15, 26B12, 39B22, 47J05, 94A08, $91 \mathrm{~B} 14$

\section{Introduction and motivation}

Given a finite collection of maps $\left\{f_{1}, f_{2}, \ldots, f_{n}\right\}$ from a set $X$ into another set $Y$, a new map $f_{n+1}: X \rightarrow Y$ obtained someway from the given ones

\footnotetext{
${ }^{1}$ Corresponding author: Esteban Induráin. Phone: +34-948169551. Fax: +34948166057. e-mail address: steiner@unavarra.es. 
$f_{1}, f_{2}, \ldots, f_{n}$ is usually said to be an aggregation of those maps. A typical example is the arithmetic mean $f_{n+1}=\frac{f_{1}+\ldots+f_{n}}{n}$ of $n$ real-valued functions (here $Y=\mathbb{R}$, the real line).

Since information fusion appears in almost every application, aggregation has become a crucial technique and it has generated a wide spread research field (see, for example $[8,9,15]$ ). From an applied point of view, aggregation functions have also been used for solving real-world problems, for example in fuzzy set theory $([3,13])$ or image processing $([12,38,26])$.

Having these ideas in mind, we wonder which is the information we need in order to obtain the aggregating map $f_{n+1}$. Given an element $x \in X$, to compute $f_{n+1}(x)$ sometimes we should know the maps $f_{1}, \ldots, f_{n}$ as a whole, maybe in all the points of its domain, or at least in several points different from $x$. But it may also happen that in order to get $f_{n+1}(x)$ we only need to have at hand the values of $f_{1}, \ldots, f_{n}$ at that point $x$. That is: $f_{n+1}(x)$ directly comes from $f_{1}(x), \ldots, f_{n}(x)$, and we may then assume that there exists a map $G: Y^{n} \rightarrow Y$ such that $f_{n+1}(x)=G\left(f_{1}(x), \ldots, f_{n}(x)\right)$ holds true for every $x \in X$. Then we say that $f_{n+1}$ depends pointwise on the collection $\left\{f_{1}, f_{2}, \ldots, f_{n}\right\}$.

Let us consider the following illustrative example:

Example 1. Let $f_{1}, f_{2}: \mathbb{R} \rightarrow \mathbb{R}$ denote two real valued functions on one single real variable. Let $f_{3}, f_{4}: \mathbb{R} \rightarrow \mathbb{R}$ respectively be defined, for every $x \in \mathbb{R}$, through the functional equations $f_{3}(x)=f_{1}(x)+f_{2}(x)$ and $f_{4}(x)=$ $f_{1}(2 x)+f_{2}(3 x)$. Despite both functional equations looking similar at first glance, from a structural point of view they are quite different. The reason is that, working with the former one, in order to know the value of the map $f_{3}$ at a point $x \in \mathbb{R}$ we only need to know the values that $f_{1}$ and $f_{2}$ take at the same point $x$. However, in the second equation, to determine $f_{4}(x)$ it is not enough to know the values of $f_{1}(x)$ and $f_{2}(x)$. As a matter of fact, we need to know the values of $f_{1}$ and $f_{2}$ at point(s) different from $x$.

This nuance is essential in our approach throughout the present paper. The first case corresponds to the so-called pointwise aggregation of maps. The second case, despite still corresponding to aggregation of maps, cannot be called "pointwise", at least a priori.

In the present paper, given two abstract (nonempty) sets $X, Y$ as well as a natural number $n$ and $n$ maps $\left\{f_{1}, f_{2}, \ldots, f_{n}: X \rightarrow Y\right\}$, we study how to aggregate those maps to obtain a new one, say, $f_{n+1}: X \rightarrow Y$ in a way such that the value of $f_{n+1}$ at a point $x \in X$ depends only on the 
values $\left\{f_{1}(x), f_{2}(x), \ldots, f_{n}(x)\right\}$ at the same point $x$. This is what we call $a$ pointwise aggregation of $\left\{f_{1}, \ldots, f_{n}\right\}$.

The structure of the paper goes as follows:

In Section 2 we formalize the notion of pointwise aggregation of maps. In Section 3 we study the main structural functional equation linked to the pointwise aggregations of maps. The particular case of real-valued functions is studied in Section 4. In Section 5 we analyze a relevant case study, namely type-2 fuzzy sets via fusion operators.

In Section 6 we discuss several applications and uses in social choice theory. We conclude with a final Section 7 that includes suggestions for further research.

\section{Previous concepts and notation}

Definition 1. Let $X, Y$ denote two (nonempty) sets. Let $n$ be a natural number. Let $Y^{X}$ denote the set of maps from $X$ into $Y$, that is, $Y^{X}=\{f$ : $X \rightarrow Y\}$. Let $\left(f_{1}, \ldots, f_{n}\right) \in\left(Y^{X}\right)^{n}$ stand for an $n$-tuple of maps from $X$ into $Y$. A map $f_{n+1} \in Y^{X}$ is said to be:

(i) An aggregation of $\left(f_{1}, \ldots, f_{n}\right)$ if there exists a map $T:\left(Y^{X}\right)^{n} \rightarrow Y^{X}$ such that $f_{n+1}=T\left(f_{1}, \ldots, f_{n}\right)$. In this case the map $T$ is said to be an $n$-dimensional aggregation operator. ${ }^{2}$.

(ii) A pointwise aggregation of $\left(f_{1}, \ldots, f_{n}\right)$ if there exists a map $W: Y^{n} \rightarrow$ $Y$ such that $f_{n+1}(x)=W\left(f_{1}(x), \ldots, f_{n}(x)\right)$ holds for every $x \in X$. In this case, the map $W$ is said to be a pointwise $n$-dimensional aggregator, whereas the functional equation $f_{n+1}(x)=W\left(f_{1}(x), \ldots, f_{n}(x)\right)$ is said to be the structural functional equation of pointwise aggregation of maps.

\footnotetext{
${ }^{2}$ Notice that our definition of " $n$-dimensional aggregation operator" is less restrictive than the usual ones that are often encountered in the literature of fuzzy sets and related topics (see e.g. [15, 9]). We have decided to keep it for the sake of completeness, bearing in mind that possible applications in other settings -perhaps unrelated to fuzzy set theory- could also come from this more general Definition 1. (See e.g. the applications in mathematical social choice theory analyzed in Section 6 in the present manuscript).
} 
Remark 1. (i) When $n=1$, we use the word modifier instead of aggregator. It is implicitly understood that when we aggregate maps, at least two maps are involved in the process.

(ii) In general it is not true that an aggregation $f_{n+1}$ of $n$ maps $f_{1}, \ldots, f_{n}$ from a set $X$ into another set $Y$ is actually a pointwise aggregation (see the Example 2 below).

(iii) In general, the order of the elements $f_{1}, \ldots, f_{n}$ in the $n$-tuple is relevant for the description of the problem.

Example 2. Let $X=Y=\mathbb{N}$ be the set of natural numbers, and let $f_{1}$ : $\mathbb{N} \rightarrow \mathbb{N}$ defined as $f_{1}(1)=f_{1}(3)=0$, and $f_{1}(n)=n$ otherwise. Consider the equation $f_{2}(n)=f_{1}(2 n)$, for every $n \in \mathbb{N}$. Then $f_{2}$ is a modifier of $f_{1}$, but it is not a pointwise modifier of $f_{1}$. Indeed, assume that there is $W: \mathbb{N} \rightarrow \mathbb{N}$ such that $f_{2}(n)=W\left(f_{1}(n)\right)$ holds true for every $n \in N$. Then, on the one side, we would have $W(0)=W\left(f_{1}(1)\right)=f_{2}(1)=f_{1}(2)=2$. On the other side, we would obtain $W(0)=W\left(f_{1}(3)\right)=f_{2}(3)=f_{1}(6)=6$, reaching a contradiction.

Definition 2. Let $X, Y$ be two nonempty sets. Let $T:\left(Y^{X}\right)^{n} \rightarrow Y^{X}$ denote an $n$-dimensional aggregation operator of maps from $X$ into $Y$. Then $T$ is said to be:

representable if there is a map $W: Y^{n} \rightarrow Y$ such that $T\left(f_{1}, \ldots, f_{n}\right)(x)=$ $W\left(f_{1}(x), \ldots, f_{n}(x)\right)$ holds for every $x \in X$ and every $n$-tuple $\left(f_{1}, \ldots, f_{n}\right) \in$ $\left(Y^{X}\right)^{n}$.

Example 3. In general, an $n$-dimensional aggregation operator from a set $X$ into another set $Y$ may fail to be representable. To see this, let $X=Y=\mathbb{R}$ be the real line, and consider any bijection $f: \mathbb{R} \rightarrow \mathbb{R}$. It is clear that given a real number $x \in \mathbb{R}$, the value $f(x)$ has no relationship (in general) with the value $f(-x)$. In other words: despite knowing $x$ and $f(x)$, we cannot guess the value $f(-x)$. Therefore, the modifier $T$ defined by $T(f)(x)=f(-x)$ for every $x \in \mathbb{R}$ and every function $f: \mathbb{R} \rightarrow \mathbb{R}$ is not representable.

Remark 2. (i) The concept of a representable aggregation operator has been considered in [19] for the particular case $X$ finite and $Y=\mathbb{R}$, and in [20], for an arbitrary set $X$ and $Y=\mathbb{R}$.

(ii) Given a representable $n$-dimensional aggregation operator $T:\left(Y^{X}\right)^{n} \rightarrow$ $Y^{X}$, we immediately observe that the functional equation that naturally 
arises as $T\left(f_{1}, \ldots, f_{n}\right)(x)=W\left(f_{1}(x), \ldots, f_{n}(x)\right)$ has a solution $W$ that only depends on the given operator $T$. In particular, for any fixed $n$-tuple of functions $\left.\left(f_{1}, \ldots, f_{n}\right) \in\left(Y^{X}\right)^{n}\right)$, by calling $f_{n+1}=T\left(f_{1}, \ldots, f_{n}\right)$, it follows that the map $W$ functional equation of pointwise aggregation of maps.

\section{Representability of operators and the structural functional equa- tion associated to pointwise aggregation of maps}

Given two nonempty sets $X$ and $Y$, our aim is to characterize the representable $n$-dimensional aggregation operators $T:\left(Y^{X}\right)^{n} \rightarrow Y^{X}$. In other words, we are interested in finding the solutions (if any) of the structural functional equation $T\left(f_{1}, \ldots, f_{n}\right)(x)=W\left(f_{1}(x), \ldots, f_{n}(x)\right)$ with $x \in X$ and $\left(f_{1}, \ldots, f_{n}\right) \in\left(Y^{X}\right)^{n}$, paying attention to those solutions that only depend on $T$.

Remark 3. Observe that, conceptually, the problem of solving a structural functional equation that corresponds to pointwise aggregation of maps, namely $f_{n+1}(x)=W\left(f_{1}(x), \ldots, f_{n}(x)\right)$ for every $x \in X$, in which all the maps $f_{1}, \ldots, f_{n}, f_{n+1}$ are already known a priori, is totally different from, starting from an $n$-dimensional aggregation operator $T$ and setting $T\left(f_{1}, \ldots, f_{n}\right)=$ $f_{n+1}$ for every $n$-tuple of maps $\left(f_{1}, \ldots, f_{n}\right) \in\left(Y^{X}\right)^{n}$, searching for a map $W: Y^{n} \rightarrow Y$ such that $f_{n+1}(x)=W\left(f_{1}(x), \ldots, f_{n}(x)\right)$ holds for every $x \in X$ and every $\left(f_{1}, \ldots, f_{n}\right) \in\left(Y^{X}\right)^{n}$. That is: in this second situation the maps $f_{1}, \ldots, f_{n}$ are generic, and obviously not given a priori, so that $W$ only depends on $T$.

Nevertheless, it is also important to notice that if $W$ is a solution of a structural functional equation of pointwise aggregation, we may try to study if $W$ gives rise to an $n$-dimensional operator $T$ such that $f_{n+1}(x)=$ $W\left(f_{1}(x), \ldots, f_{n}(x)\right)$ for every $x \in X$, independently of $f_{1}, \ldots, f_{n}$, where $f_{n+1}$ denotes $T\left(f_{1}, \ldots, f_{n}\right)$. When this happens, $T$ is representable.

We are now ready to introduce some characterizations of the representability of $n$-dimensional aggregation operators from a set $X$ into another set $Y$. To do so, first we introduce a definition.

Definition 3. Let $X, Y$ be two nonempty sets. Let $T:\left(Y^{X}\right)^{n} \rightarrow Y^{X}$ be an $n$-dimensional aggregation operator. Then $T$ is said to be:

(i) fully independent if it holds that $\left(f_{1}(x), \ldots, f_{n}(x)\right)=\left(g_{1}(t), \ldots g_{n}(t)\right) \Rightarrow$ $T\left(f_{1}, \ldots, f_{n}\right)(x)=T\left(g_{1}, \ldots, g_{n}\right)(t)$, for every $x, t \in X$ and $\left(f_{1}, \ldots, f_{n}\right)$, $\left(g_{1}, \ldots, g_{n}\right) \in\left(Y^{X}\right)^{n}$. 
(ii) independent as regards maps if $\left(f_{1}(x), \ldots, f_{n}(x)\right)=\left(g_{1}(x), \ldots g_{n}(x)\right) \Rightarrow$ $T\left(f_{1}, \ldots, f_{n}\right)(x)=T\left(g_{1}, \ldots, g_{n}\right)(x)$ for every $x \in X$ and $\left(f_{1}, \ldots, f_{n}\right)$, $\left(g_{1}, \ldots, g_{n}\right) \in\left(Y^{X}\right)^{n}$.

(iii) pointwise independent whenever $\left(f_{1}(x), \ldots, f_{n}(x)\right)=\left(f_{1}(t), \ldots f_{n}(t)\right) \Rightarrow$ $T\left(f_{1}, \ldots, f_{n}\right)(x)=T\left(f_{1}, \ldots, f_{n}\right)(t)$ for every $x, t \in X$ and $\left(f_{1}, \ldots, f_{n}\right) \in$ $\left(Y^{X}\right)^{n}$.

The next result generalizes Theorem 3.4 of [20].

Theorem 1. Let $X, Y$ be two nonempty sets. Let $T:\left(Y^{X}\right)^{n} \rightarrow Y^{X}$ be an $n$ dimensional aggregation operator. The following statements are equivalent:

(i) $T$ is representable,

(ii) $T$ is fully independent,

(iii) $T$ is independent as regards maps, and pointwise independent.

Proof. The implications (i) $\Rightarrow$ (ii) $\Rightarrow$ (iii) are straightforward. To prove (iii) $\Rightarrow(\mathrm{i})$, let $\left(y_{1}, \ldots, y_{n}\right) \in Y^{n}$. Let $c_{y_{i}}: X \rightarrow Y$ be the constant map defined by $c_{y_{i}}(x)=y_{i}$ for every $x \in X(i=1, \ldots, n)$. Fix an element $x_{0} \in X$. Define now $W: Y^{n} \rightarrow Y$ as $W\left(y_{1}, \ldots, y_{n}\right)=T\left(c_{y_{1}}, \ldots, c_{y_{n}}\right)\left(x_{0}\right)$. Observe that the choice of $x_{0}$ is irrelevant here, since $T$ is pointwise independent. In order to see that $W$ represents $T$, fix $x \in X$ and $\left(f_{1}, \ldots, f_{n}\right) \in\left(Y^{X}\right)^{n}$. Let $c_{i}: X \rightarrow Y$ be the constant map given by $c_{i}(t)=f_{i}(x)$ for every $t \in X(i=1, \ldots, n)$. Since $T$ is independent as regards maps, it follows that $T\left(f_{1}, \ldots, f_{n}\right)(x)=T\left(c_{1}, \ldots, c_{n}\right)(x)$. But, by definition of $W$, we also have that $T\left(c_{1}, \ldots, c_{n}\right)(x)=W\left(f_{1}(x), \ldots, f_{n}(x)\right)$. Therefore $T\left(f_{1}, \ldots, f_{n}\right)(x)=$ $W\left(f_{1}(x), \ldots, f_{n}(x)\right)$ and we are done.

For the particular case of modifiers of maps from a set $X$ into itself, we obtain the following corollary.

Corollary 1. Let $X$ be a nonempty set and let $T: X^{X} \rightarrow X^{X}$ be a modifier. If $T$ is representable, then the map $W=T\left(1_{X}\right) \in X^{X}$, where $1_{X}$ denotes the identity map, is actually a representation of $T$.

Proof. Notice that $T$ is fully independent by Theorem 1. Thus, given $t \in X$ and $f \in X^{X}$, we have that $f \circ 1_{X}(t)=f(t)=1_{X}(f(t))$. Hence $T\left(1_{X}\right)(f(t))=$ $T\left(f \circ 1_{X}\right)(t)=T(f)(t)$. Therefore $W(f(t))=T\left(1_{X}\right)(f(t))=T\left(f \circ 1_{X}\right)(t)=$ $T(f)(t)$ holds for every $t \in X$, so that $T$ is representable by means of $W$. 
Example 4. Let $X, Y$ be two nonempty sets. The following $n$-dimensional aggregation operators from $X$ into $Y$ are obviously representable:

(i) each projection $\pi_{i}:\left(Y^{X}\right)^{n} \rightarrow Y^{X}$, where $\pi_{i}\left(f_{1}, \ldots, f_{n}\right)=f_{i}$ for every $\left(f_{1}, \ldots, f_{n}\right) \in\left(Y^{X}\right)^{n}(i=1, \ldots, n)$,

(ii) each constant operator mapping any $n$-tuple $\left(f_{1}, \ldots, f_{n}\right) \in\left(Y^{X}\right)^{n}$ to a (fixed a priori) map $g: X \rightarrow Y$.

Moreover, any $n$-ary operation in $Y$ immediately gives rise to a representable $n$-dimensional aggregation operator from $X$ into $Y$. Indeed, given a map $H: Y^{n} \rightarrow Y$, it is clear that the $n$-dimensional aggregation operator $T_{H}:\left(Y^{X}\right)^{n} \rightarrow Y^{X}$ given by $T_{H}\left(f_{1}, \ldots, f_{n}\right)(x)=H\left(f_{1}(x), \ldots, f_{n}(x)\right)$ for every $x \in X$ and $\left(f_{1}, \ldots, f_{n}\right) \in\left(Y^{X}\right)^{n}$ is representable through $H$.

Remark 4. Despite the operators mentioned in Example 4 (i) above being a trivial case of representable aggregation operators, they play a crucial role in many contexts coming from miscellaneous applications where it is important to detect those operators that are actually projections. Thus, in many contexts that study systems of voting or ranking of objects, arising in social choice theory, it is usual to consider a set $Y$ of $m$ elements called alternatives. Assuming that each voter ranks these alternatives by means of a linear order (also known as a profile) that reflects her/his individual preferences, we have at hand a set of $n$ maps from $X=\{1, \ldots, m\}$ to $Y$. (Here $n$ is the number of voters). If $f_{i}$ is the map that corresponds to the agent $i(i=1, \ldots, n)$, we shall understand that she/he prefers the alternative $f_{i}(1)$ as her/his best one, $f_{i}(2)$ as her/his second best and so on. In this context, a social rule is a map from $\left(Y^{X}\right)^{n}$ to $\left(Y^{X}\right)$, that tries to aggregate the individual preferences into a social one, so defining a new ranking (a linear order or a social profile) on the set of alternatives, based on the individual profiles.

In order to obtain some suitable social ranking, that is, one that preserves (in some sense) the individual preferences, several classical models arising in social choice try to impose common sense restrictions to the social rules. However, in most of these models, the unique possible social rules are the so-called dictatorial ones, namely, the projections. (See e.g. [4, 5, 27, 41, 42, 30, 39] for a further account). We discuss some applications in this direction later on, in Section 6.

In the spirit of Definition 3 and Theorem 1 we finish this section by considering that an $n$-tuple of functions from a set $X$ into another set $Y$ has 
been fixed. We furnish a result concerning the structural functional equation of pointwise aggregation.

Theorem 2. Let $X, Y$ be two nonempty sets. Let $\left(f_{1}, \ldots, f_{n}\right) \in\left(Y^{X}\right)^{n}$ denote a fixed $n$-tuple of maps from $X$ into $Y$. Let $f_{n+1}: X \rightarrow Y$ be a map. The following statements are equivalent:

(i) There exists a solution $W: Y^{n} \rightarrow Y$ of the structural functional equation of pointwise aggregation so that $f_{n+1}(x)=W\left(f_{1}(x), \ldots, f_{n}(x)\right)$ holds for every $x \in X$.

(ii) The implication $\left(f_{1}(x), \ldots, f_{n}(x)\right)=\left(f_{1}(t), \ldots f_{n}(t)\right) \Rightarrow f_{n+1}(x)=$ $f_{n+1}(t)$ holds true for all $x, t \in X$.

Proof. The implication (i) $\Rightarrow$ (ii) is obvious. To prove that (ii) $\Rightarrow$ (i), we choose an element $y_{0} \in Y$, and define $W$ as follows: given $\left(y_{1}, \ldots y_{n}\right) \in Y^{n}$ we declare that $W\left(y_{1}, \ldots y_{n}\right)=f_{n+1}(x)$ if there exists $x \in X$ such that $\left(y_{1}, \ldots y_{n}\right)=\left(f_{1}(x), \ldots f_{n}(x)\right)$; otherwise, $W\left(y_{1}, \ldots y_{n}\right)=y_{0}$.

\section{Pointwise aggregation of real-valued functions}

As we have seen in Example 4, given two nonempty sets $X$ and $Y$ the set of representable $n$-dimensional aggregation operators from $X$ into $Y$ could be too big. Consequently, we do not try to give a list of all the representable $n$ dimensional aggregation operators from a set $X$ into another set $Y$. Not even we intend to describe general solutions of the structural functional equation of pointwise aggregation. Particular results, paying an special attention to the aggregation of real-valued functions, or at least, maps from a set $X$ into another set $Y$ such that either $X$ or $Y$ (or both) is the real line $\mathbb{R}$.

To start with, we analyze some invariance properties that are closely related to the representability of $n$-dimensional aggregation operators from a set $X$ into the real line $\mathbb{R}$. To do so, first we introduce some previous definitions.

Definition 4. Let $A$ denote a nonempty set. Each element of $A^{A}$, the set of all maps from $A$ into itself, is said to be a transformation of the set $A$.

Definition 5. Let $X, Y$ denote two nonempty sets. Let $T:\left(Y^{X}\right)^{n} \rightarrow Y^{X}$ be an $n$-dimensional aggregation operator. Let $\phi_{1}, \ldots, \phi_{n}, \gamma: Y \rightarrow Y$ stand for $n+1$ (fixed) transformations of $Y$. Let $g: X \rightarrow Y$ be a (fixed) map from $X$ into $Y$. Then the operator $T$ is said to be: 
(i) $g$-constant if for any $n$-tuple of maps $\left(f_{1}, \ldots, f_{n}\right) \in\left(Y^{X}\right)^{n}$ it holds that $T\left(f_{1}, \ldots, f_{n}\right)=g$.

(ii) strongly constant (or trivial) if it is $g$-constant such that the corresponding map $g: X \rightarrow Y$ is also a constant map, that is, there exists $y_{0} \in Y$ with $g(x)=y_{0}$ for every $x \in X$. (In other words: $T\left(f_{1}, \ldots, f_{n}\right)(x)=y_{0}$ for every $\left(f_{1}, \ldots, f_{n}\right) \in\left(Y^{X}\right)^{n}$ and every $\left.x \in X\right)$.

(iii) $\left(\phi_{1}, \ldots, \phi_{n}, \gamma\right)$-invariant if for every $n$-tuple $\left(f_{1}, \ldots, f_{n}\right) \in\left(Y^{X}\right)^{n}$ it holds that $T\left(\phi_{1} \circ f_{1}, \ldots, \phi_{n} \circ f_{n}\right)=\gamma \circ T\left(f_{1}, \ldots, f_{n}\right)$.

In the same way, if $F, G$ are two (fixed) nonempty subsets of the set $Y^{Y}$ of transformations of $Y$, the operator $T$ is said to be:

(iv) $(F, G)$-invariant if for every $\left\{\phi_{1}, \ldots, \phi_{n}\right\} \subseteq F$ and $\left(f_{1}, \ldots, f_{n}\right) \in\left(Y^{X}\right)^{n}$ there is a map $\gamma \in G$, which depends on $\left\{\phi_{1}, \ldots, \phi_{n}\right\}$ and $\left(f_{1}, \ldots, f_{n}\right)$, such that $T\left(\phi_{1} \circ f_{1}, \ldots, \phi_{n} \circ f_{n}\right)=\gamma \circ T\left(f_{1}, \ldots, f_{n}\right)$.

Remark 5. It should be noted that the concept of invariance as defined in [20] is slightly different from ours (see also Definition 1(2) in [19] for the particular case $X$ finite).

Definition 6. Let $Y$ denote a nonempty set. A subset $F \subseteq Y^{Y}$ of transformations of $Y$ is said to be stable if it satisfies the following properties:

(a) Each map $f \in F$ is a bijection whose inverse $f^{-1}$ also belongs to the set $F$.

(b) The identity map $1_{Y}$ (given by $1_{Y}(t)=t$ for every $t \in Y$ ) belongs to $F$.

(c) The subset $F$ is closed under composition of maps, that is $g \circ f \in F$, for every $f, g \in F$.

Example 5. Let $\mathbb{R}$ be the real line. The collection $\mathcal{I}$ of strictly increasing functions from $\mathbb{R}$ into $\mathbb{R}$ is stable. In addition, the collection $\mathcal{A}$ of positively affine functions from $\mathbb{R}$ into $\mathbb{R}$, given by $\mathcal{A}=\left\{f \in \mathbb{R}^{\mathbb{R}}\right.$ : there exists $a>0$ and $b \in \mathbb{R}$ such that $f(x)=a x+b$ for every $x \in \mathbb{R}\}$ is stable, too.

Definition 7. Let $X, Y$ denote two nonempty sets. Let $F \subseteq Y^{Y}$ be a stable subset of transformations of $Y$. Given two $n$-tuples $\left(f_{1}, \ldots, f_{n}\right)$ and $\left(g_{1}, \ldots, g_{n}\right) \in\left(Y^{X}\right)^{n}$ of maps from $X$ to $Y$, we say that $\left(f_{1}, \ldots, f_{n}\right)$ and $\left(g_{1}, \ldots, g_{n}\right)$ are $F$-equivalent if there exist $n$ maps $\phi_{1}, \ldots, \phi_{n} \in F$ such that $\left(g_{1}, \ldots, g_{n}\right)=\left(\phi_{1} \circ f_{1}, \ldots, \phi_{n} \circ f_{n}\right)$. 
Remark 6. Notice that by Definition 6, the relationship of $F$-equivalence just defined is indeed an equivalence relation on $\left(Y^{X}\right)^{n}$.

Moreover, comparing with Definition 5 , we observe that an $n$-dimensional aggregation operator $T \in\left(Y^{X}\right)^{n}$ is $(F, G)$-invariant if and only if for every $\left(f_{1}, \ldots, f_{n}\right)$ and $\left(g_{1}, \ldots, g_{n}\right) \in\left(Y^{X}\right)^{n}$ that are $F$-equivalent, it holds that $T\left(f_{1}, \ldots, f_{n}\right)$ and $T\left(g_{1}, \ldots, g_{n}\right) \in Y^{X}$ are $G$-equivalent.

We are ready now to introduce a result that links pointwise aggregation to invariance properties. ${ }^{3}$

Theorem 3. Let $X$ be a set with at least two points and let $T:\left(\mathbb{R}^{X}\right)^{n} \rightarrow \mathbb{R}^{X}$ be an $n$-dimensional aggregation operator. Let $\mathcal{A}$ (respectively, $\mathcal{I}$ ) denote the set of all positively affine (respectively, strictly increasing) transformations from $\mathbb{R}$ into $\mathbb{R}$. The following statements are equivalent:

(i) The aggregation operator $T$ is $(\mathcal{A}, \mathcal{I})$-invariant and representable,

(ii) Either $T$ is a strongly constant operator, or there exists $j \in\{1, \ldots, n\}$ and a strictly monotone (i.e.: either strictly increasing or strictly decreasing) function $g: \mathbb{R} \rightarrow \mathbb{R}$ such that $T\left(f_{1}, \ldots, f_{n}\right)=g \circ f_{j}$, for every $\left(f_{1}, \ldots, f_{n}\right) \in\left(\mathbb{R}^{X}\right)^{n}$.

Proof. The implication $(i i) \Rightarrow(i)$ is straightforward. Therefore, we only prove the fact $(i) \Rightarrow(i i)$.

To do so, first we observe that by the representability of $T$ there exists a function $W: \mathbb{R}^{n} \rightarrow \mathbb{R}$ such that $T\left(f_{1}, \ldots, f_{n}\right)(x)=W\left(f_{1}(x), \ldots, f_{n}(x)\right)$ holds for every $\left(f_{1}, \ldots, f_{n}\right) \in\left(\mathbb{R}^{X}\right)^{n}$ and every $x \in X$.

Let us see that the function $W$ satisfies that:

$W\left(u_{1}, \ldots, u_{n}\right) \leq W\left(v_{1}, \ldots, v_{n}\right) \Leftrightarrow W\left(a_{1} u_{1}+b_{1}, \ldots, a_{n} u_{n}+b_{n}\right) \leq W\left(a_{1} v_{1}+\right.$ $\left.b_{1}, \ldots, a_{n} v_{n}+b_{n}\right)$ for any $\left(u_{1}, \ldots, u_{n}\right),\left(v_{1}, \ldots, v_{n}\right),\left(b_{1}, \ldots, b_{n}\right) \in \mathbb{R}^{n}$ and $\left(a_{1}, \ldots, a_{n}\right) \in(0,+\infty)^{n}$.

Indeed, the converse implication is obvious by taking $a_{1}=\ldots=a_{n}=1$ and $b_{1}=\ldots=b_{n}=0$. In order to prove the direct implication, let $\left(u_{1}, \ldots, u_{n}\right),\left(v_{1}, \ldots, v_{n}\right), \quad\left(b_{1}, \ldots, b_{n}\right) \in \mathbb{R}^{n}$ and $\left(a_{1}, \ldots, a_{n}\right) \in(0,+\infty)^{n}$ be fixed. Take now $\left(f_{1}, \ldots, f_{n}\right) \in\left(\mathbb{R}^{X}\right)^{n}$ and $x, y \in X$ such that, for each

\footnotetext{
${ }^{3}$ In addition, in [20] the concept of a comparison meaningful, with respect to independent ordinal scales, aggregation operator is studied. For the particular case of real-valued functions of $n$ variables, this concept turns out to be a fundamental axiom in measurement theory (see $[33,34]$ for further details).
} 
$i \in\{1, \ldots, n\}, f_{i}(x)=u_{i}$ and $f_{i}(y)=v_{i}$. Notice that, since we are assuming that the cardinality of $X$ is strictly bigger than 1, this is always possible. So, $W\left(u_{1}, \ldots, u_{n}\right)=T\left(f_{1}, \ldots, f_{n}\right)(x) \leq T\left(f_{1}, \ldots, f_{n}\right)(y)=W\left(v_{1}, \ldots, v_{n}\right)$. Consider, for each $i \in\{1, \ldots, n\}$, the transformation $\phi_{i}(t)=a_{i} t+b_{i} \in \mathcal{A}$. Then, by $(\mathcal{A}, \mathcal{I})$-invariance, there is a strictly increasing transformation, say $\gamma \in \mathcal{I}$, such that $T\left(\phi_{1} \circ f_{1}, \ldots, \phi_{n} \circ f_{n}\right)=\gamma \circ T\left(f_{1}, \ldots, f_{n}\right)$. So, $T\left(\phi_{1} \circ f_{1}, \ldots, \phi_{n} \circ f_{n}\right)(x)=\gamma \circ T\left(f_{1}, \ldots, f_{n}\right)(x)=\gamma\left(T\left(f_{1}, \ldots, f_{n}\right)(x)\right) \leq$ $\gamma\left(T\left(f_{1}, \ldots, f_{n}\right)(y)\right)=\gamma \circ T\left(f_{1}, \ldots, f_{n}\right)(y)=T\left(\phi_{1} \circ f_{1}, \ldots, \phi_{n} \circ f_{n}\right)(y)$.

Now, for each $z \in X$, it holds that $T\left(\phi_{1} \circ f_{1}, \ldots, \phi_{n} \circ f_{n}\right)(z)=W\left(\phi_{1} \circ\right.$ $\left.f_{1}(z), \ldots, \phi_{n} \circ f_{n}(z)\right)=W\left(a_{1} f_{1}(z)+b_{1}, \ldots, a_{n} f_{n}(z)+b_{n}\right)$. Thus, $W\left(a_{1} f_{1}(x)+\right.$ $\left.b_{1}, \ldots, a_{n} f_{n}(x)+b_{n}\right)=T\left(\phi_{1} \circ f_{1}, \ldots, \phi_{n} \circ f_{n}\right)(x) \leq T\left(\phi_{1} \circ f_{1}, \ldots, \phi_{n} \circ f_{n}\right)(y)=$ $W\left(a_{1} f_{1}(y)+b_{1}, \ldots, a_{n} f_{n}(y)+b_{n}\right)$, and the inequality is proved.

Let us see now that this last fact implies that either $W: \mathbb{R}^{n} \rightarrow \mathbb{R}$ is a constant function, or, alternatively, there exist both $j \in\{1, \ldots n\}$ and a strictly monotonic function $g: \mathbb{R} \rightarrow \mathbb{R}$ such that $W\left(t_{1}, \ldots, t_{n}\right)=g\left(t_{j}\right)$ holds true for every $n$-tuple $\left(t_{1}, \ldots, t_{n}\right) \in \mathbb{R}^{n}$. To that end, consider the total preorder, say $\precsim_{W}$, on $\mathbb{R}^{n}$ defined by $W$ as follows: $\left(s_{1}, \ldots, s_{n}\right) \precsim_{W}$ $\left(t_{1}, \ldots, t_{n}\right) \Leftrightarrow W\left(s_{1}, \ldots, s_{n}\right) \leq W\left(s_{1}, \ldots, s_{n}\right)$.

By the properties of $W$, using Theorem 2 in [31] as well as Theorem 3 and its subsequent results in [18], it follows that either $\precsim_{W}$ is trivial (and consequently $W$ is a constant function), or $\precsim_{W}$ is non-trivial and, in this case, either there is $j \in\{1, \ldots, n\}$ such that $\left(s_{1}, \ldots, s_{n}\right) \precsim_{W}\left(t_{1}, \ldots, t_{n}\right) \Leftrightarrow s_{j} \leq t_{j}$ holds for every $\left(s_{1}, \ldots, s_{n}\right),\left(t_{1}, \ldots t_{n}\right) \in \mathbb{R}^{n}$, or, in a dual way, there exists $j \in\{1, \ldots, n\}$ such that $\left(s_{1}, \ldots, s_{n}\right) \precsim_{W}\left(t_{1}, \ldots, t_{n}\right) \Leftrightarrow t_{j} \leq s_{j}$ holds for any $\left(s_{1}, \ldots, s_{n}\right),\left(t_{1}, \ldots t_{n}\right) \in \mathbb{R}^{n}$.

Therefore, in the former of these two last situations in which $\precsim_{W}$ is nontrivial, there exists a strictly increasing function $g: \mathbb{R} \rightarrow \mathbb{R}$ such that $W=g \circ$ $\pi_{j}$, where $\pi_{j}: \mathbb{R}^{n} \rightarrow \mathbb{R}$ stands here for the projection on the $j$-th component, that is, $\pi_{j}\left(t_{1}, \ldots, t_{n}\right)=t_{j}$ for every $n$-tuple $\left(t_{1}, \ldots, t_{n}\right) \in \mathbb{R}^{n}$.

And in the second of these two situations, where $\precsim_{W}$ is non-trivial, there exists a strictly decreasing function $h: \mathbb{R} \rightarrow \mathbb{R}$ such that $W=h \circ \pi_{j}$.

The proof concludes immediately, by just taking into account that the operator $T$ is representable through $W$.

Remark 7. If the cardinality of $X$ is $1, \mathbb{R}^{X}$ can be identified with $\mathbb{R}$. Thus, an aggregation operator $T:\left(\mathbb{R}^{X}\right)^{n} \rightarrow \mathbb{R}^{X}$ clearly reduces to a function from $\mathbb{R}^{n}$ into $\mathbb{R}$. Therefore, in this case, any aggregation operator is trivially representable. 
Moreover, $(\mathcal{A}, \mathcal{I})$-invariance is also trivially met when $X$ is a singleton. So, in this case, the statement reads as follows:

$<<$ For a function $T: \mathbb{R}^{n} \rightarrow \mathbb{R}$ the following assertions are equivalent:

(1) $T\left(u_{1}, \ldots, u_{n}\right) \leq T\left(v_{1}, \ldots, v_{n}\right) \Leftrightarrow T\left(a_{1} u_{1}+b_{1}, \ldots, a_{n} u_{n}+b_{n}\right) \leq T\left(a_{1} v_{1}+\right.$ $\left.b_{1}, \ldots, a_{n} v_{n}+b_{n}\right)$ holds true, for any $n$-tuples $\left(u_{1}, \ldots, u_{n}\right),\left(v_{1}, \ldots, v_{n}\right)$, $\left(b_{1}, \ldots, b_{n}\right) \in \mathbb{R}^{n}$ and $\left(a_{1}, \ldots, a_{n}\right) \in(0,+\infty)^{n}$,

(2) Either $T$ is a constant function, or there exists $j \in\{1, \ldots, n\}$ and a strictly monotone function $g: \mathbb{R} \rightarrow \mathbb{R}$ such that $T\left(u_{1}, \ldots, u_{n}\right)=g\left(u_{j}\right)$, for every $\left(u_{1}, \ldots, u_{n}\right) \in \mathbb{R}^{n} .>>$

An immediate consequence of Theorem 3 appears when the operator $T$ satisfies a property of idempotence (see Definition 8 below). As a matter of fact, the subsequent Corollary 2 characterizes projections among $n$ dimensional operators from a set $X$, whose cardinality is strictly bigger than 1 , to the real line $Y=\mathbb{R}$.

Definition 8. Let $X, Y$ be two nonempty sets. An $n$-dimensional operator $T:\left(Y^{X}\right)^{n} \rightarrow Y^{X}$ is said to be idempotent if for every map $f \in Y^{X}$ it holds that $T(f, \ldots, f)=f$.

Corollary 2. Let $T:\left(\mathbb{R}^{X}\right)^{n} \rightarrow \mathbb{R}^{X}$ be an $n$-dimensional aggregation operator, where $X$ has, at least, two points. The following statements are equivalent:

(i) The operator $T$ is $(\mathcal{A}, \mathcal{I})$-invariant, idempotent and representable,

(ii) The operator $T$ is a projection: there exists $j \in\{1, \ldots, n\}$ such that $T\left(f_{1}, \ldots, f_{n}\right)=f_{j}$, for every $\left(f_{1}, \ldots, f_{n}\right) \in\left(\mathbb{R}^{X}\right)^{n}$.

Remark 8. The assumption of $X$ having at least two points cannot be dropped from the statement of Corollary 2. Indeed, if $X$ is a singleton, then, as already mentioned in Remark $7(\mathrm{v})$, an aggregation operator $T$ is a function from $\mathbb{R}^{n}$ into $\mathbb{R}$. Note that the function $T: \mathbb{R}^{n} \rightarrow \mathbb{R}$ defined as $T\left(u_{1}, \ldots, u_{n}\right)=\max \left(u_{i}\right)$, for every $\left(u_{1}, \ldots, u_{n}\right) \in \mathbb{R}^{n}, i \in\{1, \ldots, n\}$, is $(\mathcal{A}, \mathcal{I})$-invariant, idempotent and representable. Nevertheless, it is not a projection. A version of Corollary 2, in the case that $X$ is a singleton, can be obtained by adding to the idempotence property the fulfillment of the condition $W\left(u_{1}, \ldots, u_{n}\right) \leq W\left(v_{1}, \ldots, v_{n}\right) \Leftrightarrow W\left(a_{1} u_{1}+b_{1}, \ldots, a_{n} u_{n}+b_{n}\right) \leq$ $W\left(a_{1} v_{1}+b_{1}, \ldots, a_{n} v_{n}+b_{n}\right)$ for any $\left(u_{1}, \ldots, u_{n}\right),\left(v_{1}, \ldots, v_{n}\right),\left(b_{1}, \ldots, b_{n}\right) \in \mathbb{R}^{n}$ and $\left(a_{1}, \ldots, a_{n}\right) \in(0,+\infty)^{n}$. 


\section{A case study: type-2 fuzzy sets via fusion operators}

\subsection{Fusion of type-2 fuzzy sets}

Since Zadeh introduced the concept of a fuzzy set in 1965 [44], the interest for the different possible extensions of such sets has been increasingly growing, both from a theoretical and from an applied point of view [14]. Among the extensions of fuzzy sets some of the most relevant ones are the interval-valued fuzzy sets [40], the Atanassov's intuitionistic fuzzy sets [6] and the type-2 fuzzy sets [45], which encompass the two previous ones. In this work we focus on the latter, having in mind the results introduced in Section 2 and Section 3. and with an eye kept in the application in fields such as

As an extension of the concept of a fusion operator relative to fuzzy sets (functions that take $m$ values (membership degrees) in $[0,1]$ and give back a new value in $[0,1])$, and taking into account that the membership values of the elements in a type-2 fuzzy set are given in terms of new fuzzy sets over the universe $[0,1]$ (that is, by means of functions defined over $[0,1]$ ) we define fusion operators for type-2 fuzzy sets as mappings that take $m$ functions from $[0,1]$ to $[0,1]$ into a new function in the same domain. That is, functions of the type $F:\left([0,1]^{[0,1]}\right)^{m} \rightarrow[0,1]^{[0,1]}$. Our goal is to study these functions in a way as general as possible, since in principle no restriction is imposed to the membership values of a type- 2 fuzzy set. So we do not require a priori any property such as continuity, monotonicity, symmetry, etc. (See also [17] for more details).

In this subsection we recall the concepts of a fuzzy set and a type-2 fuzzy set. From here on we denote by $X$ a nonempty set that represents the universe of discourse.

Definition 9. A fuzzy set $A$ on the universe $X$ is defined as the graph of a function $\mu_{A}: X \longrightarrow[0,1]$. Therefore, $A=\left\{\left(x, \mu_{A}(x)\right) \mid x \in X\right\} \subset X \times[0,1]$. The function $\mu_{A}$ is said to be the membership function (also known as the indicator) of $A$ with respect to the universe $X$. Given $x \in X$, the value $\mu_{A}(x) \in[0,1]$ is called the membership degree of the element $x$ as regards $X$.

In [45], Zadeh introduced the concept of a type-2 fuzzy set as a generalization of a fuzzy set (also called type-1 fuzzy set). In type- 2 fuzzy sets, the corresponding notion of the membership degree of an element with respect to the universe considered is given by a fuzzy set whose universe is again $[0,1]$. That is, the membership degree of an element to a type-2 fuzzy set becomes 
now a function that belongs to $[0,1]^{[0,1]}$, the set of all possible functions from $[0,1]$ to $[0,1]$. The mathematical formalization of the notion of type-2 fuzzy set was made in $[23,37]$.

Definition 10. A type-2 fuzzy set $A$ on the universe $X$ is defined as $A=$ $\left\{\left(x, \mu_{A}(x)\right) \mid x \in X\right\}$, where $\mu_{A}: X \rightarrow[0,1]^{[0,1]}$.

As a particular case of Definition 1 and Definition 2 the following concepts are introduced in this context.

Definition 11. Let $\left(f_{1}, \ldots, f_{m}\right) \in\left([0,1]^{[0,1]}\right)^{m}$ stand for a $m$-tuple of maps from $[0,1]$ into $[0,1]$.

(i) A map $f_{m+1} \in[0,1]^{[0,1]}$ is said to be a fusion of $\left(f_{1}, \ldots, f_{m}\right)$ if there exists a map $F:\left([0,1]^{[0,1]}\right)^{m} \rightarrow[0,1]^{[0,1]}$ such that $f_{m+1}=F\left(f_{1}, \ldots, f_{m}\right)$. In this case the map $F$ is said to be an $m$-dimensional fusion operator.

(ii) A map $f_{m+1} \in[0,1]^{[0,1]}$ is said to be a pointwise fusion of $\left(f_{1}, \ldots, f_{m}\right)$ if there exists a function $W:[0,1]^{m} \rightarrow[0,1]$ satisfying that $f_{m+1}(x)=$ $W\left(f_{1}(x), \ldots, f_{m}(x)\right)$ for every $x \in[0,1]$. In this case, the map $W$ is said to be a pointwise m-dimensional fusion operator, whereas the functional equation $f_{m+1}(x)=W\left(f_{1}(x), \ldots, f_{m}(x)\right)$ is said to be the structural functional equation of pointwise fusion operators.

(iii) Let $F:\left([0,1]^{[0,1]}\right)^{m} \rightarrow[0,1]^{[0,1]}$ denote an $m$-dimensional fusion operator of maps from $[0,1]$ into $[0,1]$. Then $F$ is said to be representable if there is a map $W:[0,1]^{n} \rightarrow[0,1]$ such that $F\left(f_{1}, \ldots, f_{m}\right)(x)=$ $W\left(f_{1}(x), \ldots, f_{m}(x)\right)$ holds true for every $x \in[0,1]$ and $\left(f_{1}, \ldots, f_{m}\right) \in$ $\left([0,1]^{[0,1]}\right)^{m}$.

Remark 9. Obviously Definition 3 and the subsequent results (Theorem 1 , etc.) stated in Section 2 can also be used in this setting, now working with type-2 fuzzy sets and fusion operators.

\subsection{Union and intersection of type-2 fuzzy sets as fusion operators}

In this subsection we focus on two key concepts to deal with type-2 fuzzy sets, namely the union and the intersection. Recall that the union and intersection of two type-2 fuzzy sets is a new type- 2 fuzzy set. Therefore, we can interpret the union and intersection of type- 2 fuzzy sets as a special case of fusion of type-2 fuzzy sets. 
It is important to say that there does not exist a unique definition for union and intersection of type-2 fuzzy sets. However, the operations considered in this work cover several cases since they act in the same way. For each element in the universe $X$, we use a function that fuses the membership functions of that element to each set. So these operations can be seen as fusion operators $F:\left([0,1]^{[0,1]}\right)^{2} \rightarrow[0,1]^{[0,1]}$.

Considering type-2 fuzzy sets as a special case of $L$-fuzzy sets launched by Goguen [28], the union and intersection of type-2 fuzzy sets is stated leaning on the union and intersection of fuzzy sets, as follows [29].

Definition 12. Let $f_{1}, f_{2} \in[0,1]^{[0,1]}$ be two functions. The operations (respectively called union and intersection $) \cup, \cap:\left([0,1]^{[0,1]}\right)^{2} \rightarrow[0,1]^{[0,1]}$ are respectively defined as $\left(f_{1} \cup f_{2}\right)(x)=\max \left(f_{1}(x), f_{2}(x)\right)$ and $\left(f_{1} \cap f_{2}\right)(x)=$ $\min \left(f_{1}(x), f_{2}(x)\right)$ for every $x$ in the unit interval $[0,1]$.

The following result is straightforward.

Proposition 1. The mappings $\cup, \cap:\left([0,1]^{[0,1]}\right)^{2} \rightarrow[0,1]^{[0,1]}$ are representable fusion operators for all $f_{1}, f_{2} \in[0,1]^{[0,1]}$.

The problem with the previous definition of union and intersection of type-2 fuzzy sets is that these concepts do not retrieve the usual union and intersection of fuzzy sets [24]. In order to avoid this trouble, another definition of union and intersection of type-2 fuzzy sets was introduced in this literature, based on Zadeh's extension principle [37, 24, 35, 43].

Definition 13. Let $f_{1}, f_{2} \in[0,1]^{[0,1]}$ be two maps. The operations (again, respectively called union and intersection, but in the sense of Zadeh's extension principle) $\sqcup, \sqcap:\left([0,1]^{[0,1]}\right)^{2} \rightarrow[0,1]^{[0,1]}$ are respectively defined as $\left(f_{1} \sqcup f_{2}\right)(x)=\sup \left\{\left(f_{1}(y) \wedge f_{2}(z)\right): y \vee z=x\right\}$ and $\left(f_{1} \sqcap f_{2}\right)(x)=$ $\sup \left\{\left(f_{1}(y) \wedge f_{2}(z)\right): y \wedge z=x\right\}$ for every $x \in[0,1]$.

(Here, given $a, b \in[0,1]$, the standard latticial notation goes as follows: $a \vee b=\max \{a, b\}$, whereas $a \wedge b=\min \{a, b\})$.

Remark 10. Observe that the fusion operators $\sqcup$ and $\sqcap$ are completely different from $\cup$ and $\cap$. In general, for any $f_{1}, f_{2} \in[0,1]^{[0,1]}$ it is not possible to know the values $\left(f_{1} \sqcup f_{2}\right)(x)$ and $\left(f_{1} \sqcap f_{2}\right)(x)$ by knowing only the values of $f_{1}(x)$ and $f_{2}(x)$.

Proposition 2. In general, the mappings $\sqcup, \sqcap:\left([0,1]^{[0,1]}\right)^{2} \rightarrow[0,1]^{[0,1]}$ fail to be representable fusion operators. 
Nevertheless, we may still notice that the union and intersection of fuzzy sets (in the sense of Zadeh's extension principle) indeed become pointwise fusion operators on a suitable (smaller) restricted domain.

Theorem 4. The following statements hold true:

(i) If $f_{1}, f_{2} \in[0,1]^{[0,1]}$ are increasing mappings then the operation $\sqcup$ is a pointwise fusion operator.

(ii) If $f_{1}, f_{2} \in[0,1]^{[0,1]}$ are decreasing mappings then the operation $\square$ is a pointwise fusion operator.

Proof. (See also [43])

(i) For the case $\sqcup$, let us see that $\{(y, z) \mid y \vee z=x\}=\{(x, z) \mid z \leq x\} \cup$ $\{(y, x) \mid y \leq x\}$

In the first situation, namely for $\{(x, z) \mid z \leq x\}$, since the function $f_{2}$ is increasing, we get

$f_{2}(z) \leq f_{2}(x)$ for all $z \leq x$. So, $f_{1}(x) \wedge f_{2}(z) \leq f_{1}(x) \wedge f_{2}(x)$ for all $z \leq x$. In particular, $\sup _{z \leq x}\left\{f_{1}(x) \wedge f_{2}(z)\right\} \leq f_{1}(x) \wedge f_{2}(x)$. Moreover, since the point $(x, x)$ lies in the set considered, we have that $\sup _{z \leq x}\left\{f_{1}(x) \wedge f_{2}(z)\right\}=$ $f_{1}(x) \wedge f_{2}(x)$.

In a similar way, in the second situation, for $\{(y, x) \mid y \leq x\}$, it holds that $\sup _{y \leq x}\left\{f_{1}(y) \wedge f_{2}(x)\right\}=f_{1}(x) \wedge f_{2}(x)$.

Therefore $\left(f_{1} \sqcup f_{2}\right)(x)=\sup \left\{\left(f_{1}(y) \wedge f_{2}(z): y \vee z=x\right\}=\right.$ $\vee\left(\sup _{z \leq x}\left\{f_{1}(x) \wedge f_{2}(z)\right\}, \sup _{y \leq x}\left\{f_{1}(y) \wedge f_{2}(x)\right\}\right)=f_{1}(x) \wedge f_{2}(x)$.

Hence the union is a pointwise fusion operator.

(ii) This case, for the intersection $\Pi$, is handled in an entirely analogous way to the case $\sqcup$ just discussed.

\section{Some applications and uses in social choice theory}

\subsection{Columns vs. rows in the Arrowian setting}

Let $X=\{1, \ldots, m\}$ be the set of the first $m$ natural numbers. A map $f: X \rightarrow X$ can be described through a column vector of $m$ elements such that the $i$-th entry of this column vector is $f(i) \in X$, with $i$ varying from 1 to $m$. If we have at hand $n$ such maps, say $\left\{f_{1}, \ldots, f_{n}\right\}$ from $X$ into $X$, then we can jointly represent them by a $m \times n$ matrix whose entry in the $i$-th row and $j$-th column is $f_{j}(i)$. Here $i \in\{1, \ldots, m\}$ whereas $j \in\{1, \ldots, n\}$. A $n$-dimensional operator $T:\left(X^{X}\right)^{n} \rightarrow X^{X}$ can be understood as a rule that 
operates with the columns $\left\{f_{1}, \ldots, f_{n}\right\}$ of each of those matrices $m \times n$ to accordingly get a new column vector $T\left(f_{1}, \ldots, f_{n}\right) \in X^{X}$.

However, when $T$ is representable, due to the satisfaction of the structural functional equation of pointwise aggregation, there exist a map $W$ : $X^{n} \rightarrow X$ such that $T\left(f_{1}, \ldots, f_{n}\right)(i)=W\left(f_{1}(i), \ldots, f_{n}(i)\right)$ holds for every $i \in\{1, \ldots m\}$. This means that we can get the column vector $T\left(f_{1}, \ldots, f_{n}\right)$ working directly on each row of the $m \times n$ matrix that defines $T$.

All this has important uses in voting theory.

In fact, as already pointed out in Remark 4, the famous Arrow's impossibility theorem in social choice theory (see e.g. [4, 5, 27, 30, 42, 39, 41, 32]) deals with conditions on the rankings of preferences that $n$ agents define on $m$ objects, in a way that each individual ranks the $m$ objects by means of a linear (total) order, thus defining a column vector that represents her/his preferences.

Therefore, in this procedure there are $n$ such columns, so that column $j$ where $j$ varies from 1 to $n$ reflects the preferences of the individual $j$. In these contexts, the preferences of the agents are usually defined through permutations (that is, bijective maps) stated on the given set of $m$ objects or alternatives. Consequently, we can visualize all the preference rankings established by the $n$ agents using a $m \times n$ matrix, in such a way that each column corresponds to an individual preference, as remarked before.

A social choice aggregation rule (in the Arrowian sense) is then an $n$ dimensional aggregation operator that acts over the columns of such a matrix in order to define a social ranking, also given by means of a suitable permutation on the set of $m$ objects. In addition, some conditions of, say, common sense are assumed to be satisfied by the aggregation operator ${ }^{4}$.

Kenneth J. Arrow established in $[4,5]$ a set of conditions, all of them being quite natural, that, perhaps surprisingly, imply that the only possible social preference that accomplishes all such conditions of aggregation is one of the columns of the given $m \times n$ matrix, so that the social preference is indeed a projection: it must coincide with the preference of at least one of the $m$ agents, who could be considered as a dictator in the social choice theory context and terminology. (See also [42]).

\footnotetext{
${ }^{4}$ To put an obvious example, when all the columns of the matrix are identical, or, in other words, provided that all the agents have defined exactly the same ranking, the social preference should compulsorily agree with such columns, thus respecting the unanimity.
} 
Thus, Arrow's theorem is based on vertical conditions (i.e., conditions on the individual profiles that correspond to columns of the matrix) imposed on the aggregation rules.

However, as already studied e.g. in [39], sometimes it is possible to achieve similar results, now based on horizontal aggregation conditions (i.e., conditions on the rows of the matrix), expressed in terms of the absolute position of an alternative in a preference ranking. As a matter of fact, it is possible to obtain a positional version of Arrow's theorem for the case when the possibility of indifference is removed (so that preferences are always strict), see [39] for further details.

This last possibility obviously links with the results introduced throughout the present manuscript. Indeed, if a social rule, say $T$, is defined through a representable $n$-dimensional aggregation operator (not necessarily being a projection), it is then obvious that it can be re-obtained working by rows instead of by columns, due to the satisfaction of the structural functional equation of pointwise agregation.

\subsection{Social evaluation operators}

Now we consider a social choice model that takes as primitive the concept of a social evaluation functional. Basically, a social evaluation functional is an aggregation operator that maps a $n$-tuple of (individual) real-valued functions defined on an unstructured set $X$ into a (social) real-valued function defined on $X$. (See $[10,36]$ for an excellent account of the role played by utility methods in social choice theory. See also $[2,1,16]$ to find some other functional equations related to utility theory and/or social choice. For further details on social evaluation functionals, see [19]).

Let $X$ be a nonempty set (usually called the set of alternatives) and let $n \geq 1$ be a natural number (number of agents). A typical function, from $X$ into $\mathbb{R}$, corresponding to the agent $j$, is denoted by $f_{j}$. As in the previous sections, the set of all real-valued functions from $X$ into $\mathbb{R}$ is denoted by $\mathbb{R}^{X}$.

A typical $n$-tuple of real-valued functions is denoted by $F=\left(f_{1}, \ldots, f_{n}\right)$ and $\left(\mathbb{R}^{X}\right)^{n}$ stands for the set of all those $n$-tuples.

For a given $x \in X$ and $F=\left(f_{1}, \ldots, f_{n}\right) \in\left(\mathbb{R}^{X}\right)^{n}, F(x)$ denotes the $n$-tuple $\left(f_{1}(x), \ldots f_{n}(x)\right) \in \mathbb{R}^{n}$.

Let $\mathcal{A}$ stand for the set of positively affine maps from $\mathbb{R}^{n}$ into $\mathbb{R}^{n}$. Given $n$-tuples $a=\left(a_{1}, \ldots, a_{n}\right) \in(0,+\infty)^{n}$ and $b=\left(b_{1}, \ldots, b_{n}\right) \in \mathbb{R}^{n}$ a typical

element (that we denote by $A_{b}^{a}$ ) of the set $\mathcal{A}$ satisfies that $A_{b}^{a}\left(x_{1}, \ldots, x_{n}\right)=$ $\left(a_{1} x_{1}+b_{1}, \ldots, a_{n} x_{n}+b_{n}\right)$, for every $\left(x_{1}, \ldots, x_{n}\right) \in \mathbb{R}^{n}$. 
Given $A_{b}^{a} \in \mathcal{A}$ and $F=\left(f_{1}, \ldots, f_{n}\right) \in\left(\mathbb{R}^{X}\right)^{n}$, let $A_{b}^{a} F \in\left(\mathbb{R}^{X}\right)^{n}$ denote the $n$-tuple of maps such that $A_{b}^{a} F(x)=\left(a_{1} f_{1}(x)+b_{1}, \ldots, a_{n} f_{n}(x)+b_{n}\right)$, for every $x \in X$.

Definition 14. A social evaluation functional is an $n$-dimensional aggregation operator $T:\left(\mathbb{R}^{X}\right)^{n} \rightarrow \mathbb{R}^{X}$ that associates a real-valued function $T\left(f_{1}, \ldots, f_{n}\right) \in \mathbb{R}^{X}$ to any $n$-tuple $F=\left(f_{1}, \ldots, f_{n}\right)$ in the domain $\left(\mathbb{R}^{X}\right)^{n}$. It should be noticed that, since the domain of a social evaluation functional is $\left(\mathbb{R}^{X}\right)^{n}$, the so-called property of universality of domain is implicitly assumed. A social evaluation functional $T$ is said to be trivial if there exists a constant real-valued map $f_{0} \in \mathbb{R}^{X}$ such that $T\left(f_{1}, \ldots, f_{n}\right)=f_{0}$, for all $\left(f_{1}, \ldots, f_{n}\right) \in\left(\mathbb{R}^{X}\right)^{n}$ and $f_{0}(t)=r_{0} \in \mathbb{R}$, for every $t \in X$ (here $r_{0}$ is the constant value that $f_{0}$ takes on $X$ ). In other words, the aggregation operator $T$ is strongly constant in the sense of Definition 5 (ii).

We now present a basic definition that essentially translates the usual welfarist axioms involved in the definition of a social welfare functional to the setting of social evaluation functionals.

Definition 15. A social evaluation functional $T:\left(\mathbb{R}^{X}\right)^{n} \rightarrow \mathbb{R}^{X}$ is said to satisfy:

(1) the property of welfarism if there is a function $W: \mathbb{R}^{n} \longrightarrow \mathbb{R}$ such that $T\left(f_{1}, \ldots, f_{n}\right)(x)=W\left(f_{1}(x), \ldots, f_{n}(x)\right)$, for every $\left(f_{1}, \ldots, f_{n}\right) \in\left(\mathbb{R}^{X}\right)^{n}$ and $x \in X$ (i.e., $T$ is representable in the sense of Definition 2 ),

(2) the property of information invariance with respect to independent interval scales if $T\left(f_{1}, \ldots, f_{n}\right)(x) \leq T\left(f_{1}, \ldots, f_{n}\right)(y) \Longleftrightarrow T\left(A_{a}^{b} F\right)(x) \leq$ $T\left(A_{a}^{b} F\right)(y)$, for every $F=\left(f_{1}, \ldots, f_{n}\right) \in\left(\mathbb{R}^{X}\right)^{n}, x, y \in X$ and $A_{a}^{b} \in \mathcal{A}$,

(3) the weak Pareto property, if for every $F=\left(f_{1}, \ldots, f_{n}\right) \in\left(\mathbb{R}^{X}\right)^{n}$ and $x, y \in X$ such that $f_{j}(x)<f_{j}(y)$ holds for every $j \in\{1, \ldots, n\}$, it holds that $T\left(f_{1}, \ldots, f_{n}\right)(x)<T\left(f_{1}, \ldots, f_{n}\right)(y)$,

(4) the Pareto property if it already satisfies the weak Pareto property and, in addition, for every $F=\left(f_{1}, \ldots, f_{n}\right) \in\left(\mathbb{R}^{X}\right)^{n}$ and $x, y \in X$ such that $f_{j}(x) \leq f_{j}(y)$ holds for every $j \in\{1, \ldots, n\}$, it holds that $T\left(f_{1}, \ldots, f_{n}\right)(x) \leq T\left(f_{1}, \ldots, f_{n}\right)(y)$,

(5) the strong Pareto property if for every $F=\left(f_{1}, \ldots, f_{n}\right) \in\left(\mathbb{R}^{X}\right)^{n}$ and $x, y \in X$ such that $f_{j}(x) \leq f_{j}(y)$ holds for every $j \in\{1, \ldots, n\}$ 
and $f_{k}(x)<f_{k}(y)$ holds for some $k \in\{1, \ldots, n\}$, then it holds that $T\left(f_{1}, \ldots, f_{n}\right)(x)<T\left(f_{1}, \ldots, f_{n}\right)(y)$,

(6) the strong dictatorship property if there is an individual $i \in\{1, \ldots, n\}$ (the strong dictator) such that $T\left(f_{1}, \ldots, f_{n}\right)(x) \leq T\left(f_{1}, \ldots, f_{n}\right)(y)$ if and only if $f_{i}(x) \leq f_{i}(y)$, for every $F=\left(f_{1}, \ldots, f_{n}\right) \in\left(\mathbb{R}^{X}\right)^{n}$ and $x, y \in X$,

(7) the inverse strong dictatorship property if there is $i \in\{1, \ldots, n\}$ (the inverse strong dictator) such that $T\left(f_{1}, \ldots, f_{n}\right)(x) \leq T\left(f_{1}, \ldots, f_{n}\right)(y)$ if and only if $f_{i}(y) \leq f_{i}(x)$, for every $F=\left(f_{1}, \ldots, f_{n}\right) \in\left(\mathbb{R}^{X}\right)^{n}$ and $x, y \in X$,

(8) the unanimity property if $T(f, \ldots, f)=f$, for every $f \in \mathbb{R}^{X}$,

(9) the anonymity property if $T\left(f_{\sigma(1)}, \ldots, f_{\sigma(n)}\right)=T\left(f_{1}, \ldots, f_{n}\right)$, for every permutation $\sigma$ of the set $\{1, \ldots, n\}$.

As a direct consequence of the ideas used in the proof of Theorem 3, the following results are reached. We only sketch the proof of Theorem 5 .

Theorem 5. Let $X$ be a set with at least two points. For a social evaluation functional $T:\left(\mathbb{R}^{X}\right)^{n} \rightarrow \mathbb{R}^{X}$, the following assertions are equivalent:

(i) $T$ satisfies welfarism and information invariance with respect to independent interval scales,

(ii) $T$ is trivial, or it satisfies strong dictatorship, or it satisfies inverse strong dictatorship.

Proof. The proof of the converse implication $(i i) \Rightarrow(i)$ is easy. Indeed, if $T$ is trivial, then it is sufficient to take $W\left(x_{1}, \ldots, x_{n}\right)=r_{0}$, for some fixed $r_{0} \in \mathbb{R}$, in order to see that the assumptions in (i) hold. If $T$ is strongly dictatorial, then take $W\left(x_{1}, \ldots, x_{n}\right)=x_{i}$, for every $\left(x_{1}, \ldots, x_{n}\right) \in \mathbb{R}^{n}, i$ being the strong dictator, and observe that all the conditions in (i) hold too. If $T$ is strongly inversely-dictatorial, then take $W\left(x_{1}, \ldots, x_{n}\right)=-x_{i}$, for every $\left(x_{1}, \ldots, x_{n}\right) \in$ $\mathbb{R}^{n}, i$ being the inverse strong dictator, to conclude. So, we concentrate on the proof of the direct implication $(i) \Rightarrow(i i)$. To that end, consider a real-valued function $W: \mathbb{R}^{n} \longrightarrow \mathbb{R}$ such that $T\left(f_{1}, \ldots, f_{n}\right)(x)=W\left(\left(f_{1}(x), \ldots, f_{n}(x)\right)\right.$, for every $\left(f_{1}, \ldots, f_{n}\right) \in\left(\mathbb{R}^{X}\right)^{n}$ and $x \in X$. Notice that $W$ exists because $T$ satisfies welfarism. Then, since $T$ also satisfies information invariance with 
respect to independent interval scales, it is straightforward to see that $W$ satisfies the key property in the proof of Theorem 3, namely, $W\left(u_{1}, \ldots, u_{n}\right) \leq$ $W\left(v_{1}, \ldots, v_{n}\right) \Leftrightarrow W\left(a_{1} u_{1}+b_{1}, \ldots, a_{n} u_{n}+b_{n}\right) \leq W\left(a_{1} v_{1}+b_{1}, \ldots, a_{n} v_{n}+b_{n}\right)$ for any $\left(u_{1}, \ldots, u_{n}\right),\left(v_{1}, \ldots, v_{n}\right),\left(b_{1}, \ldots, b_{n}\right) \in \mathbb{R}^{n}$ and $\left(a_{1}, \ldots, a_{n}\right) \in(0,+\infty)^{n}$.

So, as in the proof of Theorem 3 , we have that either $W$ is constant, or there are both $i \in\{1, \ldots, n\}$ and a strictly monotone function $g: \mathbb{R} \longrightarrow$ $\mathbb{R}$ such that $W\left(x_{1}, \ldots, x_{n}\right)=g\left(x_{i}\right)$, for every $\left(x_{1}, \ldots, x_{n}\right) \in \mathbb{R}^{n}$. If $W$ is constant, then $T$ is trivial. Otherwise, if $g$ is strictly increasing, then, for every $\left(f_{1}, \ldots, f_{n}\right)\left(\mathbb{R}^{X}\right)^{n}$ and $x, y \in X$, it follows that $T\left(f_{1}, \ldots, f_{n}\right)(x)=$ $W\left(f_{1}(x), \ldots, f_{n}(x)\right)=g\left(f_{i}(x)\right) \leq T\left(f_{1}, \ldots, f_{n}\right)(y)=W\left(f_{1}(y), \ldots, f_{n}(y)\right)=$ $g\left(f_{i}(y)\right)$ if and only if $f_{i}(x) \leq f_{i}(y)$. Therefore $T$ satisfies the property of strong dictatorship, $i$ acting as a strong dictator. Alternatively, if $g$ is strictly decreasing, then, in a similar manner, we see that $T$ satisfies the property of inverse strong-dictatorship, $i$ acting now as an inverse strong dictator. This finishes the proof.

Corollary 3. Let $X$ be a set with at least two points. For a social evaluation functional $T:\left(\mathbb{R}^{X}\right)^{n} \rightarrow \mathbb{R}^{X}$, the following assertions are equivalent:

(i) $T$ satisfies welfarism, information invariance with respect to independent interval scales and the Pareto property (respectively, the weak Pareto property).

(ii) Either $T$ is trivial, or it satisfies strong dictatorship (respectively, $T$ satisfies strong dictatorship).

Corollary 4. Let $X$ be a set with at least two points. For a social evaluation functional $T:\left(\mathbb{R}^{X}\right)^{n} \rightarrow \mathbb{R}^{X}$, the following assertions are equivalent:

(i) $T$ satisfies welfarism, information invariance with respect to independent interval scales and unanimity,

(ii) There is $i \in\{1, \ldots, n\}$ such that $T\left(f_{1}, \ldots, f_{n}\right)=f_{i}$, for all $\left(f_{1}, \ldots, f_{n}\right) \in$ $\left(\mathbb{R}^{X}\right)^{n}$.

\section{Remark 11.}

(i) Notice that, unlike the usual statements encountered in the social choice literature where it is required that the cardinality ("size") of $X$ is, at least, 3, our statements (Theorem 5 and Corollaries 3 to 4) only demand that this cardinality is, at least, 2 . In addition, $n$ is allowed to be 1 . 
(ii) In the case that the $X$ is a singleton, then a social evaluation functional $T:\left(\mathbb{R}^{X}\right)^{n} \rightarrow \mathbb{R}^{X}$, turns out to be a function from $\mathbb{R}^{n}$ into $\mathbb{R}$. In this case, both properties welfarism as well as information invariance with respect to independent interval scales are trivially met (see Remark 7 (v) and Remark 8) and, therefore, they play no role. So, in order to provide similar statements to those of Theorem 5 and Corollaries 3 to 4 , if $X$ is a singleton, we have to replace these two conditions by the fulfillment of condition (1) that appears in Remark 7 (v).

(iii) It is a direct consequence of Theorem 5 above that if $X$ has at least two elements, then a social evaluation functional that satisfies the properties of welfarism, information invariance with respect to independent interval scales and anonymity, is, a fortiori, trivial.

Using a counter-argument in the statement of Theorem 5, the following impossibility result can be achieved.

Theorem 6. Let $n>1$. For a set $X$ with at least two points no social evaluation functional exists that satisfies welfarism, information invariance with respect to independent interval scales and the strong Pareto property.

Remark 12. If $X$ is a singleton and $n>1$, then a similar statement to that of Theorem 6 above can be provided by taking into account the observation done in Remark 11 (ii).

\section{Final comments}

In this final section, we outline some ideas that could lead to a further development of the analysis made throughout this manuscript.

A suggestion could be the consideration of continuity properties of $n$ dimensional aggregation operators from a nonempty set $X$ into another set $Y$, provided that $X$ and $Y$ are endowed with suitable topologies (see e.g. [19]). By the way, the set $Y^{X}$ can also be endowed with a topology as, for instance, the classical compact-open topology (see e.g. pp. 257 and ff. in [25] for further details). Finally, on $\left(Y^{X}\right)^{n}$ we may consider the product topology (of the compact-open topology on each factor).

If we pay attention to representable operators, and depending on the topologies considered on $X$ and $Y$, the continuity of a map $W: Y^{n} \rightarrow Y$ that represents an aggregation operator $T:\left(Y^{X}\right)^{n} \rightarrow Y^{X}$ is intimately related to the continuity of $T$. 
To put an example in this direction, let $Y=\mathbb{R}$ be the real line endowed with the usual (Euclidean) topology. Let $X=\{1, \ldots, m\}$. Endow $X$ with the discrete topology. It is then clear that $Y^{X}$ can be identified with $\mathbb{R}^{m}$. Moreover, we can assume that, after this identification, the set $Y^{X}$ is also endowed with the Euclidean topology, because this topology and the compact-open topology on $Y^{X}$ agree in this case when $X$ is finite and $Y=\mathbb{R}$. Furthermore $\left(Y^{X}\right)^{n}$, endowed with the product topology, can also be identified with $\mathbb{R}^{m n}$ with the usual Euclidean topology. Notice also that $\mathbb{R}^{m n}$ can be viewed as the collection of all $m \times n$ matrices whose entries are real numbers. (Compare this with Remark 4 and the body of Section 6 above).

In this setting, the following straightforward result arises:

Theorem 7. Let $X=\{1, \ldots, m\}$, endowed with the discrete topology. Let $Y=\mathbb{R}$ the real line, endowed with the usual topology. Let $T:\left(Y^{X}\right)^{n} \rightarrow Y^{X}$ be a representable $n$-dimensional aggregation operator. Let $W: \mathbb{R}^{n} \rightarrow \mathbb{R}$ be a function that represents $T$, so that $T\left(f_{1}, \ldots, f_{n}\right)(x)=W\left(f_{1}(x), \ldots, f_{n}(x)\right)$ holds for every $\left(f_{1}, \ldots, f_{n}\right) \in\left(Y^{X}\right)^{N}$ and every $x \in X$. Identify $\left(Y^{X}\right)^{n}$ to $\mathbb{R}^{m n}$, and $Y^{X}$ to $\mathbb{R}^{m}$ and endow each of them with its respective usual Euclidean topology. Then, the operator $T$ is continuous if and only if the function $W$ is.

As in Section 6, this new topological setting would immediately have applications to social choice theory. For instance, in the topological Chichilnisky model arising in social choice theory, a nonempty set $Z$ endowed with a topology $\tau$ is considered. Provided that $n$ is a natural number, a social Chichilnisky $n$-rule is just a continuous map $F: Z^{n} \rightarrow Z$ satisfying the following two conditions:

(i) $F(z, \ldots, z)=z$ for every $z \in Z$,

(ii) $F\left(z_{\sigma_{1}}, \ldots, z_{\sigma_{n}}\right)=F\left(z_{1}, \ldots, z_{n}\right)$ for any permutation $\sigma$ acting on the set $\{1, \ldots, n\}$.

This is actually an abstract model ${ }^{5}$, so that it is (a priori) irrelevant here if each element $z \in Z$ is interpreted as an individual preference defined through

\footnotetext{
${ }^{5}$ As a matter of fact, the theoretical background of the topological Chichilnisky model had already appeared much earlier in the analysis of the so-called "topological spaces that admit a topological $n$-mean", see e.g. [7].
} 
a total order on a set of alternatives, or if it is a different kind of ordering on the set of alternatives, or even if it is just the best alternative that an individual has selected as her/his best option. All this depends on the particular context considered. Perhaps, the most remarkable feature to be pointed out at this stage is that Chichilnisky models furnish a theoretical and totally abstract setting, alternative to the Arrowian model. Moreover, depending on the particular context to be explored, either preference orderings or just best individual alternatives are understood as points of a topological space. It is important to notice here that the set $Z$ may now be infinite. It can be proved that the existence or nonexistence of a social Chichilnisky $n$-rule strongly depends on the topology which $Z$ is endowed with. For instance, when $(Z, \tau)$ is a finite cellular complex, social Chichilnisky $n$-rules do exist for every natural number $n$ if and only if the topological space $(Z, \tau)$ is contractible. (See $[16,22]$ for further details).

In the context of the present manuscript, if $X$ and $Y$ are nonempty sets, each of them endowed with a topology, we may wonder if there exist some operator from $\left(Y^{X}\right)^{n}$ into $Y^{X}$ that is representable through a continuous map $W: Y^{n} \rightarrow Y$ such that "it preserves the diagonal of $Y$ ", that is, $W(y, \ldots, y)=y$ for every $y \in Y$ and, in addition, "it is not affected by permutations", that is, $W\left(y_{\sigma_{1}}, \ldots, y_{\sigma_{n}}\right)=W\left(y_{1}, \ldots, y_{n}\right)$ for every permutation $\sigma$ of the set $\{1, \ldots, n\}$. As commented before, the topology on $Y$ may provoke the non-existence of such a map $W$ (see e.g. [21]).

As another interesting stream for further research we suggest matching the ideas of Section 6 (applications to social choice) with some sort of fuzzy approach, in the spirit of [36] and perhaps using some results introduced in Section 5 related to type- 2 fuzzy sets.

\section{Acknowledgments}

This work has been supported by the research projects ECO2012- 34828, MTM2012-37894-C02-02, MTM2015-63608-P, TIN2013-47605-P, TIN201129520 (Spain) and the Research Services of the Public University of Navarre.

Thanks are given to the editors and two anonymous referees for their valuable suggestions and comments.

\section{References}

[1] Abrísqueta J., Candeal J.C., Catalán R.G., De Miguel J.R., Induráin E.: Generalized Abel functional equations and numerical representability of 
semiorders, Publ. Math. Debrecen 78 (1-2), 557-568 (2011)

[2] Aczél J., Luce R.D., Marley A.A.J.: A functional equation arising from simultaneous utility representations, Results Math. 43, 193-197 (2003)

[3] Alcalde C., Burusco A., Bustince H., Jurío A., Sanz, A.: Evolution in time of $L$-fuzzy context sequences. Inform. Sci. 326, 202-214 (2016)

[4] Arrow K.J.: A difficulty in the concept of Social Choice, J. Polit. Econ. 58, 328-346 (1950)

[5] Arrow K.J.: Social Choice and Individual Values, Yale University Press, New Haven, CT, (1951)

[6] Atanassov K.T.: Intuitionistic fuzzy sets, Fuzzy Sets Systems 20, 87-96 (1986)

[7] Aumann G.: Über Räume mit Mittelbindungen, Math. Ann. 119, 210$215(1943)$

[8] Beliakov G., Bustince H., Calvo T.: A Practical Guide to Averaging Functions, Studies In Fuzziness and Soft Computing, Springer, Cham, (2016)

[9] Beliakov G., Pradera A., Calvo T.: Aggregation Functions: A Guide for Practitioners, Studies In Fuzziness and Soft Computing, Springer, Berlin, (2007)

[10] Bossert W., Weymark J.A.: Utility in social choice, in: Barberà S., Hammond P.J. and Seidl Ch. (eds), Handbook of Utility Theory 2, pp. 1099-1177, Kluwer Academic Publishers, Amsterdam, (2004)

[11] Bouyssou D.: Democracy and efficiency: A note on "Arrow's theorem is not a surprising result". European J. Oper. Res. 58 (3), 427-430 (1992)

[12] Bustince H., Barrenechea E., Pagola M.: Image thresholding using restricted equivalence functions and maximizing the measures of similarity. Fuzzy Sets Systems 158, 496-516 (2007)

[13] Bustince H., Fernandez J., Sanz J., Baczynski M., Mesiar R.: Construction of strong equality index from implication operators, Fuzzy Sets Systems 211, 15-33 (2013) 
[14] Bustince H., Herrera F., Montero J.: Fuzzy Sets and their extensions, Springer, Berlin (2007)

[15] Calvo T., Mayor G., Mesiar R.: Aggregation Operators: new trends and applications, Physica-Verlag, Heidelberg (2002)

[16] Campión M.J., Candeal J.C., Catalán R.G., De Miguel J.R., Induráin E.: Aggregation of preferences in crisp and fuzzy settings: functional equations leading to possibility results, Internat. J. Uncertain. Fuzziness Knowledge-Based Systems 19 (1), 89-114 (2011)

[17] Campión M.J., Candeal J.C., De Miguel L., Induráin E., Paternain D.: First approach of type-2 fuzzy sets via fusion operators, Proceedings of the IPMU 2014 Congress, Laurent A. et al. (eds.), Part III, CCIS 444, pp. 355-363, Montpéllier, France, (2014)

[18] Candeal J.C.: Invariance axioms for preferences: applications to social choice theory, Soc. Choice Welf. 41(3), 453-471 (2013)

[19] Candeal J.C.: Social evaluation functionals: a gateway to continuity in social choice, Soc. Choice Welf. 44(2), 369-388, (2015)

[20] Candeal J.C.: Aggregation operators, comparison meaningfulness and social choice, J. Math. Psych., (2016, to appear), http://dx.doi.org/10.1016/j.jmp.2015.10.005.

[21] Candeal J.C., Induráin E.: The Moebius strip and a social choice paradox, Econom. Lett. 45, 407-412 (1994)

[22] Chichilnisky G., Heal G.M.: Necessary and sufficient conditions for a resolution of the social choice paradox, J. Econom. Theory 31(1), 68-87 (1983)

[23] Dubois D., Prade H.: Operations in a fuzzy-valued logic, Inform. Control 43, 224-254 (1979)

[24] Dubois D., Prade H.: Fuzzy sets and systems: Theory and applications, Academic Press, New York (1980)

[25] Dugundji J.: Topology, Allyn \& Bacon, Boston (1966) 
[26] Galar M., Jurío A., López-Molina C., Paternain D., Sanz J., Bustince, H.: Aggregation functions to combine RGB color channels in stereo matching, Optics Express 21, 1247-1257 (2013)

[27] Gibbard A.: Manipulation of voting schemes: a general result, Econometrica 41, 587-601 (1973)

[28] Goguen J.A.: L-fuzzy sets, J. Math. Anal. Appl. 18, 145-174 (1967)

[29] Hardig J., Walker C., Walker E.: The variety generated by the truth value algebra of type-2 fuzzy sets, Fuzzy Sets Systems 161, 735-749 (2010)

[30] Kelly J.S.: Social Choice Theory, Springer Verlag, Berlin (1988)

[31] Krause U.: Essentially lexicographic aggregation, Soc. Choice Welf. 12, 233-244 (1995)

[32] Llamazares B., Peña T.: Aggregating preferences rankings with variable weights. European J. Oper. Res. 230 (2), 348-355 (2013)

[33] Marichal J.-L., Mesiar R.: A complete description of comparison meaningful aggregation functions mapping ordinal scales into an ordinal scale: a state of the art, Aequationes Math. 77(3), 207-236 (2009)

[34] Marichal J.-L., Mesiar R., Rückschlossová A.: A complete description of comparison meaningful functions, Aequationes Math. 69(3), 309-320 (2005)

[35] Mendel J.M., John R.I.: Type-2 fuzzy sets made simple, IEEE Trans. Fuzzy Syst. 10, 117-127 (2002)

[36] Mesiar R.: Fuzzy set approach to the utility, preference relations, and aggregation operators. European J. Oper. Res. 176 (1), 414-422 (2007)

[37] Mizumoto M., Tanaka K.: Some properties of fuzzy sets of type 2, Inform. Control 31, 312-340 (1976)

[38] Paternain D., Fernandez J., Bustince H., Mesiar R., Beliakov G.: Construction of image reduction operators using averaging aggregation functions, Fuzzy Sets Syst. 261, 87-111, 2015. 
[39] Quesada A.: A positional version of Arrow's theorem, J. Math. Econom. 41 (8), 1053-1059 (2005)

[40] Sambuc R.: Functions $\Phi$-Flous, Application a l'aide au Diagnostique en Pathologie Thyroidenne, Thèse Doctorat en Medicine, University of Marseille, (1975)

[41] Satterthwaite M.: Strategy-proofness and Arrow's conditions: existence and correspondence theorems for voting procedures and social welfare theorems, J. Econom. Theor. 10, 187-217 (1975)

[42] Vincke Ph.: Arrow's theorem is not a surprising result. European J. Oper. Res. 10 (1), 22-25 (1982)

[43] Walker C.L., Walker E.A.: The algebra of fuzzy truth values. Fuzzy Sets Systems 149, 309-347 (2005)

[44] Zadeh L.A.: Fuzzy Sets, Inform. Control 8, 338-353 (1965)

[45] Zadeh L.A.: Quantitative fuzzy semantics, Inform. Sci. 3, 159-176 (1971) 